University of Nebraska - Lincoln

DigitalCommons@University of Nebraska - Lincoln

1998

Electron Attachment Energies of the DNA Bases

Kayvan Aflatooni

Gordon A. Gallup

Paul Burrow

Follow this and additional works at: https://digitalcommons.unl.edu/physicsburrow

Part of the Atomic, Molecular and Optical Physics Commons

This Article is brought to you for free and open access by the Research Papers in Physics and Astronomy at DigitalCommons@University of Nebraska - Lincoln. It has been accepted for inclusion in Paul Burrow Publications by an authorized administrator of DigitalCommons@University of Nebraska - Lincoln. 


\title{
Electron Attachment Energies of the DNA Bases
}

\author{
K. Aflatooni, G. A. Gallup, and P. D. Burrow \\ Department of Physics and Astronomy, University of Nebraska-Lincoln, \\ Lincoln, Nebraska 68588-0111 \\ Corresponding author - P. D. Burrow, email pburrow1@unl.edu
}

\begin{abstract}
Injection of electrons into the empty $\pi^{\star}$ molecular orbitals of uracil and the DNA bases creates short-lived anion states whose energies have been determined by electron scattering. A common range of attachment energies into the lowest orbitals is observed in all the bases. Evidence for nuclear motion during the lifetimes of the anions is found in all the compounds except adenine. These properties of the bases as bridge sites along the $\pi$-stack of DNA, namely, the effective degeneracy of the anion energies and the strong excitation of vibration, are key parameters for theories of electron-transfer rate, some of which lead to inverse rather than exponentially decreasing bridge-length dependences.
\end{abstract}

The nature and efficiency of electron transfer along the " $\pi$-stack" of DNA remains an unresolved and fundamental problem, reinvigorated by recent experimental and theoretical results falling on both sides of the issue..$^{1-4} \mathrm{Up}$ to now, there have been no direct experimental determinations of one of the key components of this problem, namely,

Published in Journal of Physical Chemistry A 102 (1998), pp 6205-6207.

Copyright (C) 1998 American Chemical Society. Used by permission.

Submitted January 23, 1998; revised May 8, 1998; published July 9, 1998. 
the energies required to inject an electron into the lowest, normally empty, valence molecular orbitals of the DNA bases. Knowledge of these energies, determined at the ground-state geometries of the isolated neutral molecules, is essential for developing theoretical models of electron transfer in DNA. In particular, they serve as starting points for understanding energy changes due to solvation and to the role of molecular distortions associated with electron localization on the bases.

In the present work, we show that the energies required to add an electron to the four DNA bases and the RNA base uracil, under the conditions listed above, are positive. The anion states at the equilibrium geometry of the neutral molecule are temporary, that is, unstable against electron autodetachment, and make their appearance as "resonance" peaks in electron-scattering cross sections. ${ }^{5}$ Stated more properly, the vertical attachment energies (VAEs) are all >0, or in more familiar language, the vertical electron affinities are $<0$. We emphasize that our results are for electron occupation of the empty Valence orbitals and do not refer to the diffuse, weakly bound, anion states formed by trapping of an electron in the electric dipole fields of the bases. ${ }^{6}$

We find that the VAEs of thymine (T) and cytosine (C), the pyrimidines, differ by only $0.03 \mathrm{eV}$, and the VAEs of guanine $(\mathrm{G})$ and adenine (A), the purines, by only $0.08 \mathrm{eV}$. Contrary to "chemical" intuition, the vertically formed anions of the pyrimidines are more stable than those of the larger purines, by about $0.2 \mathrm{eV}$. Taking account of the Franck-Condon overlap between the neutral and anionic potential surfaces in each compound, we find that there is a range of energies common to all the bases over which electron attachment may take place. In other words, the lowest temporary anion states of the bases are, in a practical sense, degenerate. Additionally, we have observed evidence for nuclear motion of the temporary anions associated with the lowest empty molecular orbitals (LUMOs) of all the bases except adenine. This demonstrates that electron injection into these orbitals strongly excites vibrational modes of the neutral molecules.

Low-energy electron transmission spectroscopy (ETS) ${ }^{7}$ was employed in this work to observe the formation of temporary anion states. Briefly, an energy-selected electron beam is directed through a gas cell containing a sufficient density of the target compound to partially attenuate the beam. The scattered electrons are rejected at 
a retarding electrode following the collision region, and the transmitted current, comprising the unscattered electrons, is collected. A sharp dip in this current as a function of electron energy signals the presence of a peak in the scattering cross section arising from the formation of a temporary anion state. To accentuate this structure, the energy of the electrons is modulated in the collision cell with a small ac voltage and the derivative of the transmitted current with respect to energy is acquired using a synchronous detector. ${ }^{7}$ In the present work, the collision cell and an oven source containing the compounds were separately temperature controlled. The source and cell temperatures employed here were lower than those reported in past studies ${ }^{8}$ of the photoelectron spectra of the bases, and thus we believe that decomposition products are not significant. The ET spectra were not observed to change during the course of measurement. VAEs of the bases were calibrated to within $\pm 0.050 \mathrm{eV}$ by admitting $\mathrm{N}_{2}$ to the cell and referring to well-known features arising from the $\mathrm{N}_{2}^{-}$temporary anion. ${ }^{5}$

Observation of the temporary anion states associated with electron occupation of the LUMOs of the DNA bases was a significant experimental challenge because of the large electric dipole moments of these compounds. The dipole contribution to the total scattering cross section increases sharply as the electron energy decreases below $1 \mathrm{eV}$, creating a sloping background on which the resonance structure must be observed. Adjustment of the retarding potential used to reject scattered electrons is critical, and great care must be taken to avoid instrumental artifacts. ${ }^{9}$

Figure 1 displays the derivative of transmitted current as a function of electron energy ${ }^{10}$ in the DNA bases and uracil. The temporary anion signature is shown by a minimum followed at higher energy by a maximum in the derivative signal. The energies of the extrema indicate the approximate full width at half-maximum of the band over which attachment occurs. The anion energies are associated with the midpoints between the extrema, ${ }^{5}$ indicated by the vertical lines in Figure 1. The VAEs are summarized in Table 1. Significantly, the lowest anion states in U, T, C, and G exhibit additional fine structure characteristic of anion states whose lifetime is sufficient to allow nuclear motion. ${ }^{5}$ The structure is spaced at approximately $0.1-0.2 \mathrm{eV}$ but is not pronounced enough to determine accurately. Thermally excited 


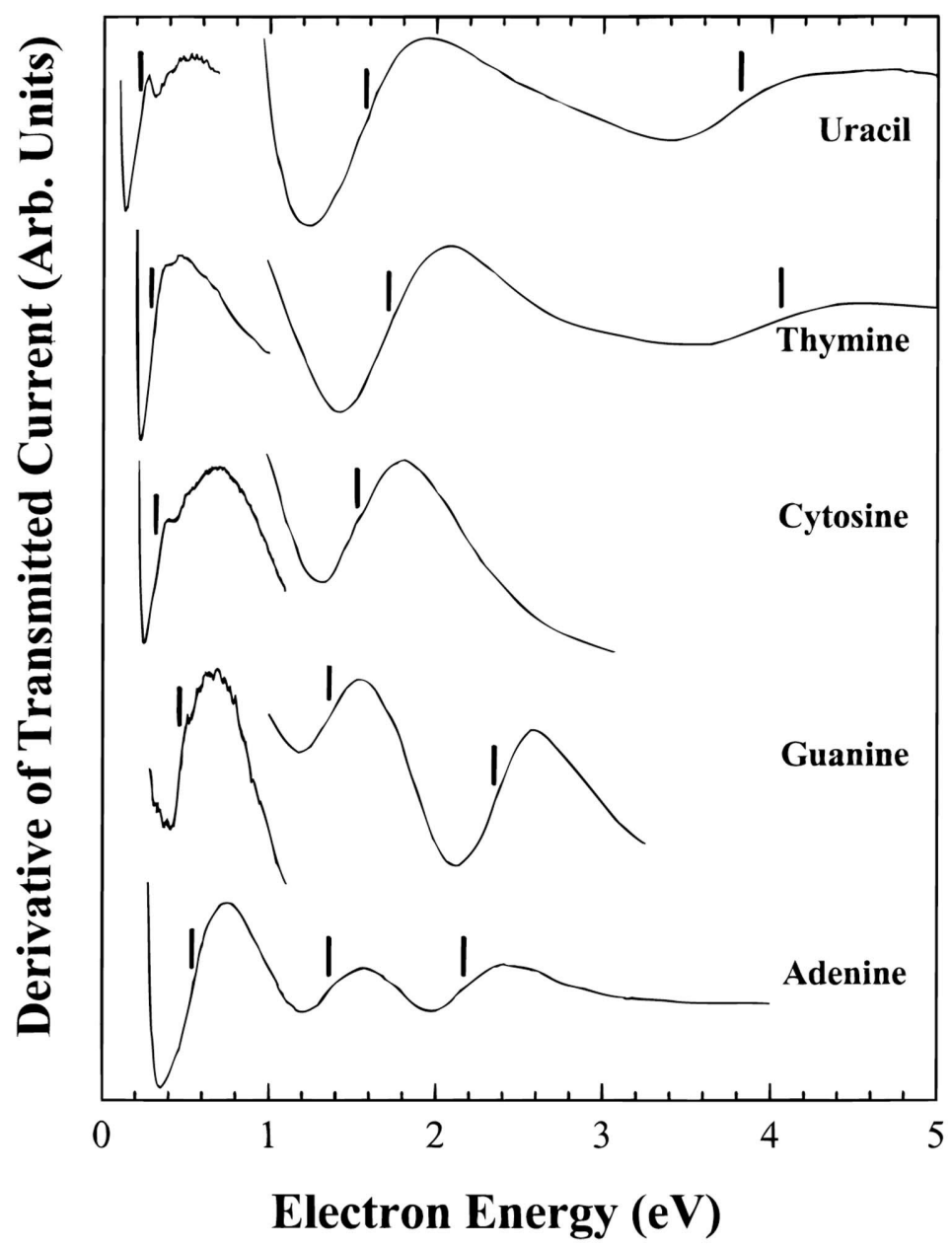

Figure 1 Derivative with respect to energy of electron current transmitted through uracil and the DNA bases, thymine, cytosine, guanine, and adenine, is plotted as a function of electron energy. The experimentally determined $\pi^{*}$ anion state energies are indicated by vertical lines.

vibrational levels of the neutral molecules undoubtedly contribute to the smearing of this structure, in addition to broadening owing to the short lifetime of the anion state.

Except for uracil, there are no other direct measurements of structure in the total scattering cross sections of these compounds for comparison. Uracil was the subject of a preliminary ETS study by A. R. Johnston and one of the present authors (P.D.B.). The results, in good agreement with those found here, have been cited elsewhere. ${ }^{11}$ 
Table 1 Experimental (ETS) and Theoretical Vertical Attachment Energies in Uracil and the DNA Bases

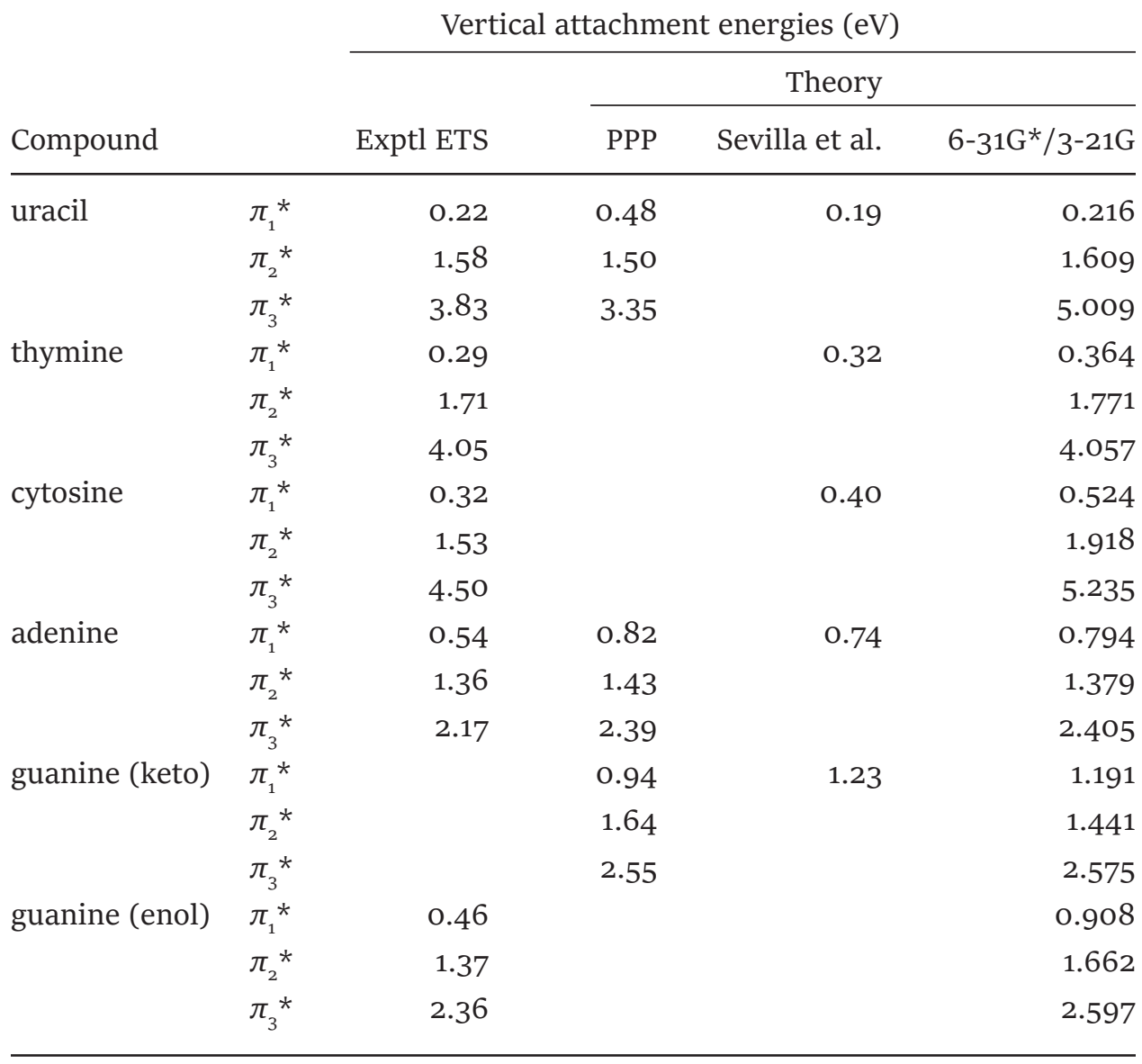

We assign the anion states observed in Figure 1 to occupation of the lowest antibonding $\pi$-orbitals in each compound. The positive sign of the VAEs is supported by the results of several theoretical studies of the bases. ${ }^{11,12}$ Although calculations of anion states that are unstable with respect to autodetachment of the electron are difficult to carry out from first principles, the problems can be circumvented with various degrees of success by use of semiempirical methods (e.g., ref 11) or ab initio calculations using restricted basis sets and approximations such as Koopmans' theorem together with empirical determination of parameters that scale the theoretical results to measured temporary anion energies in other compounds. This latter approach was used by Sevilla et al. ${ }^{12}$ who employed the experimental gas-phase VAEs of benzene, naphthalene, pyridine, and pyrimidine, as well as 
the uracil result mentioned above, to construct the set of VAEs of the bases shown in Table 1. Unfortunately, only their results for the lowest empty anion states were presented. Although the agreement is excellent for the pyrimidines, $\mathrm{U}, \mathrm{T}$, and $\mathrm{C}$, the results are less satisfactory for A and in substantial disagreement for G. Semiempirical PPP results ${ }^{11}$ are available for $\mathrm{U}, \mathrm{G}$, and $\mathrm{A}$ and are in qualitative agreement with experiment for all the anion states except for the ground-state anion of $\mathrm{G}$.

To further assure ourselves that the lowest anion states we observe are in fact the ground states, and motivated as well by the discrepancy in $\mathrm{G}$, we have carried out our own ab initio calculations ${ }^{13}$ along the lines of Sevilla et al. ${ }^{12}$ Empty orbital energies were scaled ${ }^{14}$ to known VAEs in benzene, naphthalene, pyridine, and pyrimidine. These results are also presented in Table 1 . The scaling procedure is based only on the lowest anion states of the comparison molecules and will not be reliable for high-lying states because of their mixing with core-excited anion states. Thus, we can reasonably compare results for only the lowest two anion states in $\mathrm{U}, \mathrm{T}$, and $\mathrm{C}$ and the lowest three in $\mathrm{G}$ and $\mathrm{A}$. The predicted energy differences between the first two anion states of each of the bases $\mathrm{U}, \mathrm{T}, \mathrm{C}$, and A and between the first and third states of $\mathrm{A}$ are in excellent agreement with experiment. These results further support our assertion that we have indeed observed the ground-state anions of these bases.

The present calculations in guanine, for which the keto tautomer was assumed, also indicate a significant disagreement with experiment. In particular, the spacing between the two lowest predicted anion states is much smaller than observed. The enol tautomer of guanine is an alternate form that has been observed in other environments such as rare gas matrices. ${ }^{15}$ Our calculations for the anion states of the enol tautomer are also shown in Table 1. We find that the lowest state is $0.28 \mathrm{eV}$ more stable than that of the keto tautomer and thus in somewhat better agreement with experiment. The predicted spacing between the two lowest anion states is in substantially better agreement with our measured ET spectrum. We conclude that in the gas phase and at the temperature we were using $\left(298^{\circ} \mathrm{C}\right)$, the enol tautomer is the major contributor to the spectrum.

To our knowledge, there are no direct measurements of the adiabatic electron affinities of the DNA bases in the gas phase. Wiley et 
al. ${ }^{16}$ have presented measurements of reversible reduction potentials of the bases in solution, from which they have derived gas-phase adiabatic electron affinities by "calibration" using other molecules for which both quantities are known. Their electron affinities, in eV, are C (+0.56), T (+0.79), U (+0.80), A (+0.95), and G (+1.51). These values are difficult to reconcile with the slightly negative vertical electron affinities (-VAE) obtained in the present measurement. In guanine, for example, this would indicate a stabilization of almost $2 \mathrm{eV}$ by nuclear relaxation of the anion away from the geometry of the neutral.

Returning to the results in Figure 1, we note that vertical electron attachment in each base to form the lowest anion state displays a band whose width is primarily determined by the Franck-Condon overlap of the anion potential surface with that of the neutral molecule. Electrons with energies ranging between 0.4 and $0.6 \mathrm{eV}$ will attach efficiently to any of the bases. ${ }^{17}$ The second anion states of all the bases also overlap, indicating a second set of empty orbitals whose energies are effectively degenerate.

In all of the bases except A, evidence for nuclear motion accompanying electron attachment to form the ground-state anions is observed. Although weak, the features were reproducible in all the spectra. Such fine structure is properly termed quasi-vibrational since it can appear even when the nuclei have time to move for only a single vibrational period before autodetachment is complete. ${ }^{18}$ The structure arises in one or more vibrational modes that are strongly driven by the electron charge distribution in the temporary anion. ET spectra $^{19}$ in pyridine and pyrimidine, for example, display these features in a much more pronounced fashion, where they are ascribed to totally symmetric breathing modes of the anions. Such features in the ET spectra are invariably associated with strong vibrational excitation in the neutral molecule. ${ }^{5}$

The gas-phase anionic properties of the DNA bases described in this work are entirely favorable with regard to bridge-assisted, longrange electron transfer along the $\pi$-stack of DNA. Certain of the model theories ${ }^{3,20}$ have stressed the roles of (i) near degeneracy in the energies of the bridge sites and (ii) wave function dephasing, which in the present case can arise from strong electron vibrational excitation, in achieving electron-transfer rates that fall off weakly with the number 
of bridge sites. Furthermore, our preliminary calculations of the anion state energies of two adjacent bases suggests that they are coupled by amounts comparable to vibrational energies, another favorable property.

The anion states of the bases will, of course, be stabilized by solvation. Given the low values of the VAEs, the stabilization will certainly be sufficient to prevent autodetachment of the electrons in DNA itself. Assuming that the favorable bridge properties mentioned above persist, the proximity in energy of the donor and acceptor moieties attached to the DNA to those of the bridge sites will ultimately determine the electron-transfer rates in particular experiments, as well as the most appropriate theoretical treatment. The gas-phase studies presented here therefore allow an increased level of realism in modeling such rates.

Acknowledgment This work was supported by NSF. The authors are indebted to Prof. K. D. Jordan for useful discussions and references.

\section{References and Notes}

(1) Dandliker, P. J.; Holmlin, R. E.; Barton, J. K. Science 1997, 275, 1465.

(2) Brun, A. M.; Harriman, A. J. Am. Chem. Soc. 1994, 116, 10383. Meade, T. J.; Kayyem, J. F. Angew. Chem., Int. Ed. Engl. 1995, 34, 352.

(3) Felts, A. K.; Pollard, W. T.; Friesner, R. A. J. Phys. Chem. 1995, 99, 2929.

(4) Priyadarshy, S.; Risser, S. M.; Beratan, D. N. J. Phys. Chem. 1996, 100, 17678.

(5) Schulz, G. J. Rev. Mod. Phys. 1973, 45, 378, 423. Jordan, K. D.; Burrow, P. D. Acc. Chem. Res. 1978, 11, 341.

(6) Hendricks, J. H.; Lyapustina, S. A.; de Clercq, H. L.; Snodgrass, J. T.; Bowen, K. H. J. Chem. Phys. 1996, 104, 7788. Desfrancois, C.; Addoul-Carime, H.; Schermann, J. P. J. Chem. Phys. 1996, 104, 7792. Oyler, N. A.; Adamowicz, L. Chem. Phys. Lett. 1994, 219, 223.

(7) Sanche, L.; Schulz, G. J. Phys. Rev. A 1972, 5, 1672.

(8) Padva, A.; O’Donnell, T. J.; LeBreton, P. R. Chem. Phys. Lett. 1976, 41, 278. Yu, C.; Peng, S.; Akiyama, I.; Lin, J.; LeBreton, P. R. J. Am. Chem. Soc. 1978, 10o, 2303. Lin, J.; Yu, C.; Peng, S.; Akiyama, I.; Li, K.; Lee, L. K.; LeBreton, P. R. J. Phys. Chem. 1980, 84, 1006. Lin, J.; Yu, C.; Peng, S.; Akiyama, I.; Li, K.; Lee, L. K.; LeBreton, P. R. J. Am. Chem. Soc. 1980, 102, 4627. Yu, C.; O’Donnell, T. J.; LeBreton, P. R. J. Phys. Chem. 1981, 85, 3851.

(9) Johnston, A. R.; Burrow, P. D. J. Electron Spectrosc. Relat. Phenom. 1982, 25, 119. 
(10) The discontinuity in each curve, except that of adenine, arises from using two different values of the electron rejection potential to avoid instrumental effects discussed in ref 9 . The vertical sizes of the features are not significant and were scaled for convenience of display.

(11) Compton, R. N.; Yoshioka, Y.; Jordan, K. D. Theor. Chim. Acta 1980, 54, 259. Younkin, J. M.; Smith, L. J.; Compton, R. N. Theor. Chim. Acta 1976, 41, 157.

(12) Sevilla, M. D.; Besler, B.; Colson, A.-O. J. Phys. Chem. 1995, 99, 1060.

(13) The geometries of the DNA bases were determined theoretically at the Hartree-Fock 3-21G basis level. Koopmans' theorem and the virtual orbital energies determined at the $6-31 \mathrm{G}^{*}$ basis level were used to obtain the ab initio VAEs. All the calculations were done using the GAMESS program (Schmidt, M. W.; Baldridge, K. K.; Boatz, J. A.; Elbert, S. T.; Gordon, M. S.; Jensen, J. H.; Koseki, S.; Matsunaga, N.; Nguyen, K. A.; Su, S. J.; Windus, T. L.; Dupuis, M.; Montgomery, J. A. J. Comput. Chem. 1993, 14, 1347). See also: http://www.msg.ameslab.gov/GAMESS.html

(14) VAEs (in eV) were determined from the virtual orbital energies $\left(\varepsilon_{\mathrm{vo}}\right)$ using VAE ) $\left[\varepsilon_{\mathrm{vo}}-2.5553\right] / 1.3749$. The scaling parameters were obtained using calculated orbital energies and measured ETS results from our group for benzene (VAE ) $1.12 \mathrm{eV}$ ), naphthalene (0.19), pyridine (0.59), and pyrimidine (0.38). The energy for pyrimidine is chosen at the largest vibrational feature in the ET spectrum rather than that of the lowest, since this corresponds more correctly to the VAE.

(15) Szczepaniak, K.; Szczesniak, M. J. Mol. Struct. 1987, 156, 29.

(16) Wiley, J. R.; Robinson, J. M.; Ehdaie, S.; Chen, E. C. M.; Chen, E. S. D.; Wentworth, W. E. Biochem. Biophys. Res. Commun. 1991, 180, 841.

(17) We note, however, that comparison of experiment and theory suggests that we are observing the enol tautomer of guanine. The ground-state anion of the keto form, if it is indeed $0.28 \mathrm{eV}$ less stable than that of the enol form as suggested by theory, may not exhibit quite such good overlap with the remaining bases.

(18) Birtwistle, D. T.; Herzenberg, A. J. Phys. B: At. Mol. Phys. 1971, 4, 53.

(19) Nenner, I.; Schulz, G. J. J. Chem. Phys. 1975, 62, 1747.

(20) Davis, W. B.; Wasielewski, M. R.; Ratner, M. A.; Mujica, V.; Nitzan, A. J. Phys. Chem. A 1997, 101, 6158. 